Homepage: http://epubs.icar.org.in/ejournal/index.php/JWR

\title{
Nitrogen top dressing just before irrigation improves wheat growth, productivity nitrogen use efficiency and profitability
}

Subhash Chander Gill*, Ramesh Kumar Sharma, Subhash Chandra Tripathi, Rajender Singh Chhokar, Raj Pal Meena and Ankita Jha

ICAR-Indian Institute of Wheat and Barley Research, Karnal, Haryana-132001

\section{Article history}

Received: 07 Sep., 2018

Revised : 17 Dec., 2018

Accepted: 22 Dec., 2018

\section{Citation}

Gill SC, RK Sharma, SC Tripathi, RS Chhokar, RP Meena and A Jha. 2019 Nitrogen top dressing just before irrigation improves wheat growth, productivity and nitrogen use efficiency and profitability Journal of Cereal Research 11(1):17-22 doi. org/10.25174/2249-4065/2019/83006

\section{*Corresponding author}

Email: sbhgill@yahoo.com,

(C) Society for Advancement of Wheat and Barley Research

\section{Introduction}

Wheat (Triticum aestivum L.) is the second most important staple food crop of India after rice. It is cultivated on $29.58 \mathrm{mha}$ area with $99.7 \mathrm{mt}$ production having an average yield of $3371 \mathrm{~kg} \mathrm{ha}^{-1}\left(4^{\text {th }}\right.$ advance estimates from the Agricultural Statistics Division, Directorate of Economics and Statistics, Department of Agriculture, Cooperation and Farmers Welfare, GOI, New Delhi). In India'snorthern plains, spring wheat is cultivated with the use of moderate level of $\mathrm{N}$ i.e. $150 \mathrm{~kg} \mathrm{ha}^{-1}$ (Coventry et al., 2011) and the fertilizer recommendation for wheat is also $150 \mathrm{~kg} \mathrm{~N} \mathrm{ha}^{-1}$ (2-split schedule: $1 / 3$ at sowing and $2 / 3$ at the first node stage or half at sowing and half at first irrigation) and $60 \mathrm{~kg} \mathrm{P}_{2} \mathrm{O}_{5} \mathrm{ha}^{-1}$ and $40 \mathrm{~kg} \mathrm{~K}_{2} \mathrm{O} \mathrm{ha} \mathrm{h}^{-1}$ at

\begin{abstract}
A four year field study was conducted from 2010-11 to 2013-14 at the research farm of ICAR-Indian Institute of Wheat and Barley Research, Karnal, Haryana to evaluate the influence of timing and frequency of urea top dressing on wheat productivity. The 13 treatments consisted of three nitrogen levels $(90,120$ and $150 \mathrm{~kg}$ $\mathrm{N} \mathrm{ha}^{-1}$ ), two time of application (just before applying irrigations and about a week after irrigations when it was possible to walk in the field) and two schedule of application (three equal splits as $1 / 3^{\text {rd }}$ basal, $1 / 3^{\text {rd }}$ at first irrigation and $1 / 3^{\text {rd }}$ at second irrigation; and four equal splits as $1 / 4^{\text {th }}$ basal, $1 / 4^{\text {th }}$ at first irrigation, $1 / 4^{\text {th }}$ at second irrigation and $1 / 4^{\text {th }}$ at third irrigation) along with one absolute control. The study revealed that urea top dressed just before irrigation and nitrogen splitted in three equal doses led to better crop growth, yield attributes and yield of wheat leading to improved agronomic NUE (22.5-29.8 kg grain $\mathrm{kg}^{-1}$ applied N) than urea top dressed after irrigation and nitrogen splitting in four equal doses at all levels of nitrogen application (90-150 Kg $\left.\mathrm{ha}^{-1}\right)$. The highest wheat productivity was recorded with $150 \mathrm{~kg} \mathrm{~N}$ $\mathrm{ha}^{-1}$ applied in three splits with top dressing just before irrigation. Additional yield of 2.70-5.21 $\mathrm{q} \mathrm{ha}^{-1}$ (average $3.94 \mathrm{q} \mathrm{ha}^{-1}$ ) was produced when urea was applied just before irrigation as compared to urea top dressed after irrigatin. This yield gain was 5.93 to 10.83 percent (average $8.36 \%$ ). This practice gave additional benefit of Rs. 4680 to $9043 \mathrm{ha}^{-1}$ (average Rs. $6843 \mathrm{ha}^{-1}$ ).
\end{abstract}

Keywords: Agronomic NUE, grain protein, N splitting, urea top dressing, profitability

sowing (Mishra et al., 2005; Srivastava et al., 2006). Most of the soils in Haryana and Punjab where wheat is grown in an irrigated double-cropping pattern, are deficient in $\mathrm{N}$. In the rice-wheat rotation, farmers are applying more than the recommended $\mathrm{N}$ level $\left(150 \mathrm{kgN} \mathrm{ha}^{-1}\right)$. A survey by Singh et al.(2010) indicated that farmers' in Haryana apply on an average $165.7 \mathrm{~kg} \mathrm{~N} \mathrm{ha}^{-1}$ and $30 \mathrm{~kg} \mathrm{P}_{2} \mathrm{O}_{5} \mathrm{ha}^{-1}$ to wheat with only $9.2 \%$ of the farmers used K-fertilizer. The current understanding is that a 2 -split application of $\mathrm{N}$ fertiliser is suited to the slightly heavier soils of eastern Haryana where the rice-wheat system dominates and in the west and south-west regions that have lighter soils and where rice is not grown apply $\mathrm{N}$ in 3 splits. Application of $150 \mathrm{kgN} \mathrm{ha}^{-1}$ in three splits i.e. $1 / 3$ basal at sowing, $1 / 3$ after first irrigation and $1 / 3$ at spike initiation yielded 
significantly more grain yield than $\mathrm{N}$ applied as a single application in Hisar, Haryana, a non-rice region (Bhardwaj et al., 2010).

Elsewhere in the world, $\mathrm{N}$ recoveries are higher in wheat when $\mathrm{N}$ is applied at targeted growth stages rather than all at planting, with such split applications often translating into higher yields, consistently higher grain protein and less lodging (Hobbs et al., 1998). Low $\mathrm{N}$ use efficiency due to inefficient management of fertiliser $\mathrm{N}$ is reflected in low recovery of fertiliser $\mathrm{N}$ by the crop. Nitrogen use efficiency (NUE), which is expressed as the percentage of the $\mathrm{N}$ content of the grain to the $\mathrm{N}$-fertiliser applied, illustrates how well a crop has recovered applied nitrogen fertiliser and highlight the pathways where nitrogen can be used more efficiently (Raun and Johnson, 1999; Woolfolk et al., 2002). The optimum use of $\mathrm{N}$ fertiliser will come from matching $\mathrm{N}$ supply with crop demand for $\mathrm{N}$ (Bhardwaj et al., 2010; Singh et al., 2011) and losses of $\mathrm{N}$ can be large when the $\mathrm{N}$ application is not synchronised with crop growth and development. A Bangladesh study has shown considerable opportunity to increase farmers' yields through improved $\mathrm{N}$ management without any other change in fertiliser input or crop management practices (Alam et al., 2006). Irrigation is also as important as fertiliser use, especially within the high cropping intensity areas of Haryana, in sustaining wheat productivity growth. To achieve high wheat yield, irrigation must be applied efficiently and on time. In Northern Haryana, the soils are irrigated by flooding with the water mainly drawn from underground water sources. However, many farmers also use sprinkler irrigation in SW Haryana. Irrigation is required to establish the crop and several subsequent irrigations are recommended at times corresponding to the specific growth stages (crown root initiation, early tillering, late jointing/boot, heading/flowering) of the wheat(Mishra et al., 2005). Farmers of this region are facing the problem of exact timing of fertilizer application as top dressing of urea especially at tillering (20-25DAS) and jointing (40-45 DAS) stages of crop growth and number of splits of top dressed urea whether three application $\left(1 / 3^{\text {rd }}\right.$ basal, $1 / 3^{\text {rd }}$ at tillering and $1 / 3^{\text {rd }}$ at jointing) or four split application $\left(1 / 4^{\text {th }}\right.$ basal, $1 / 4^{\text {th }}$ at tillering, $1 / 4^{\text {th }}$ at jointing and $1 / 4^{\text {th }}$ at late jointing generally coinciding with third irrigation at 60-65DAS) just before irrigation or after about a week when the field is suitable for walking. Hence, the present study was undertaken to examine the time, number of splits and rate of nitrogen in wheat crop for achieving higher productivity and nitrogen use efficiency in North Western Indian Plains.

\section{Materials and methods}

A field study was conducted to examine the time and frequency of nitrogen application especially urea top dressing on wheat productivity in Rabi season commencing from 2010-11 to 2013-14 at research farm of ICAR-Indian Institute of Wheat and Barley Research, Karnal, Haryana (Latitude $29^{\circ} 43^{\prime} \mathrm{N}$, longitude $76^{0} 58^{\prime}$ $\mathrm{E}$ and altitude $245 \mathrm{~m})$. The experimental soil was sandy loam in texture (61.4\% sand, $23.8 \%$ silt and $14.8 \%$ clay), low in organic carbon (0.30-0.35\%) and available nitrogen (169.95-179.36 $\left.\mathrm{kg} \mathrm{ha}^{-1}\right)$, medium in available phosphorus (17.09-19.87 $\left.\mathrm{kg} \mathrm{ha}^{-1}\right)$ and medium to high in available

Table1. Nitrogen levels, number of splits and time of urea top dressing details

\begin{tabular}{|c|c|c|c|}
\hline Treatment & $\begin{array}{l}\text { Nit r o g e n } \\
\text { Levels }\end{array}$ & Splits of Nitrogen & $\begin{array}{l}\text { Time of Urea top } \\
\text { dressing }\end{array}$ \\
\hline $\mathrm{T} 1$ & $90 \mathrm{~kg} \mathrm{~N} \mathrm{ha-1}$ & 3-1/3 Basal, 1/3 first irrigation, 1/3 second irrigation & After irrigation \\
\hline $\mathrm{T} 2$ & $90 \mathrm{~kg} \mathrm{~N} \mathrm{ha}^{-1}$ & 4- $1 / 4$ Basal, $1 / 4$ first irrigation, $1 / 4$ second irrigation, $1 / 4$ third irrigation & After irrigation \\
\hline $\mathrm{T} 3$ & $90 \mathrm{~kg} \mathrm{~N} \mathrm{ha-1}$ & 3-1/3 Basal, 1/3 first irrigation, 1/3 second irrigation & Before irrigation \\
\hline $\mathrm{T} 4$ & $90 \mathrm{~kg} \mathrm{~N} \mathrm{ha}^{-1}$ & 4- $1 / 4$ Basal, $1 / 4$ first irrigation, $1 / 4$ second irrigation, $1 / 4$ third irrigation & Before irrigation \\
\hline T5 & $120 \mathrm{~kg} \mathrm{~N} \mathrm{ha-1}$ & 3-1/3 Basal, 1/3 first irrigation, 1/3 second irrigation & After irrigation \\
\hline T6 & $120 \mathrm{~kg} \mathrm{~N} \mathrm{ha-1}$ & 4- $1 / 4$ Basal, $1 / 4$ first irrigation, $1 / 4$ second irrigation, $1 / 4$ third irrigation & After irrigation \\
\hline $\mathrm{T} 7$ & $120 \mathrm{~kg} \mathrm{~N} \mathrm{ha-1}$ & 3-1/3 Basal, 1/3 first irrigation, 1/3 second irrigation & Before irrigation \\
\hline $\mathrm{T} 8$ & $120 \mathrm{~kg} \mathrm{~N} \mathrm{ha-1}$ & 4- $1 / 4$ Basal, $1 / 4$ first irrigation, $1 / 4$ second irrigation, $1 / 4$ third irrigation & Before irrigation \\
\hline T9 & $150 \mathrm{~kg} \mathrm{~N} \mathrm{ha}{ }^{-1}$ & 3-1/3 Basal, 1/3 first irrigation, 1/3 second irrigation & After irrigation \\
\hline $\mathrm{T} 10$ & $150 \mathrm{~kg} \mathrm{~N} \mathrm{ha-1}$ & 4- $1 / 4$ Basal, $1 / 4$ first irrigation, $1 / 4$ second irrigation, $1 / 4$ third irrigation & After irrigation \\
\hline T11 & $150 \mathrm{~kg} \mathrm{~N} \mathrm{ha-1}$ & 3-1/3 Basal, 1/3 first irrigation, 1/3 second irrigation & Before irrigation \\
\hline $\mathrm{T} 12$ & $150 \mathrm{~kg} \mathrm{~N} \mathrm{ha-1}$ & 4- $1 / 4$ Basal, $1 / 4$ first irrigation, $1 / 4$ second irrigation, $1 / 4$ third irrigation & Before irrigation \\
\hline $\mathrm{T} 13$ & $0 \mathrm{~kg} \mathrm{~N} \mathrm{ha-1}$ & Absolute Control & \\
\hline
\end{tabular}


potash (251.68-287.52 $\left.\mathrm{kg} \mathrm{ha}^{-1}\right)$ content. The experiment was laid out in a randomized complete block design with three replications. The treatments consisted of three nitrogen levels $\left(90,120\right.$ and $\left.150 \mathrm{~kg} \mathrm{~N} \mathrm{ha}{ }^{-1}\right)$, two time of application (just before first and second irrigations and about a week after first and second irrigations depending upon the soil conditions ready to enter in the field) and two schedule of application (three equal splits as $1 / 3^{\text {rd }}$ basal, $1 / 3^{\text {rd }}$ at first irrigation at $21-25$ DAS and $1 / 3$ rd at second irrigation at $40-45 \mathrm{DAS}$; and four equal splits as $1 / 4^{\text {th }}$ basal, $1 / 4^{\text {th }}$ at first irrigation at $21-25 \mathrm{DAS}, 1 / 4^{\text {th }}$ at second irrigation at $40-45 \mathrm{DAS}$ and $1 / 4^{\text {th }}$ at third irrigation at $60-65$ DAS) along with one absolute control (no nitrogen) thus making total thirteen treatments (Table 1). The wheat was fertilized with $60 \mathrm{Kg} \mathrm{P}_{2} \mathrm{O}_{5}$ and $40 \mathrm{~kg} \mathrm{~K}_{2} \mathrm{O} \mathrm{ha}^{-1}$ as basal (at sowing) and nitrogen as per treatments. The wheat variety DBW 17 was grown at row spacing of $20 \mathrm{~cm}$ and using seed rate of $100 \mathrm{Kg} \mathrm{ha}^{-1}$ during all the four seasons. Every year the preceding crop taken was coarse rice cultivar HKR 147. The plot size for each treatment was $8.0 \mathrm{~m} \times 1.8 \mathrm{~m}$. All other agronomic practices were kept uniform for all the treatments. Data on growth and yield attributes and yield were recorded at maturity of the crop. Plant height and earhead length of five plants from each plot were measured by meter scale from the net plot area. Earhead density was recorded one meter deep from both ends in penultimate row on both sides of the every plot for one running meter, averaged and then multiplied by five to have earheads/square meter. Biomass was recorded after harvesting above ground part of matured wheat plants from net plot area after air drying. The pre-weighed bundles of wheat biomass from each plot were threshed separately by plot thresher, produce stored in cloth bags and then cleaned thoroughly before taking the final weight of grains from each plot and correcting the yield for $12 \%$ moisture. Thousand grains weight and protein content of the grains were recorded from the samples taken from each treatment at the time of final weighing of grain yield from each plot. Grains per earheads were calculated by using formula: Grains/earhead=Yield, q/ha X 10,000/ (Earheads/sq.m. x 1000 grains weight, g). Grain protein content was estimated by using NIR (Near Infra Red Reflectance) machine of FOSS make. Agronomic nitrogen use efficiency was calculated by deducting the grain yield $\left(\mathrm{kg} \mathrm{ha}^{-1}\right)$ of control treatment from the grain yield of respective treatment $\left(\mathrm{kg} \mathrm{ha}^{-1}\right)$ and dividing it by the amount of $\mathrm{N}$ applied in respective treatment $\left(\mathrm{kg} \mathrm{ha}^{-1}\right)$. Yield gain at various nitrogen levels due to pre -irrigation urea top dressing was calculated by deducting the yield of respective nitrogen level after irrigation top dressing treatment. For calculating net returns, the minimum support price of wheat was taken as Rs 1735/q.

\section{Result and discussion}

Results presented in Table 2 revealed that application of nitrogen as urea at different time and splits (3 or 4) influenced the plant height significantly during all the years as well as on pooled basis. The plant height significantly increased with nitrogen levels from 90 to $150 \mathrm{~kg} \mathrm{~N} \mathrm{ha}^{-1}$ as compared to control (no nitrogen). On pooled basis, the plant height was significantly higher in treatments where $\mathrm{N}$ was applied in three splits after irrigation as compared to respective four splits up to $120 \mathrm{~kg} \mathrm{~N} \mathrm{ha}^{-1}$.

Earhead length was also significantly affected by nitrogen levels and all the nitrogen levels recorded significantly higher earhead length than control (no nitrogen) but

Table 2. Effect of pre and post irrigation and split application of urea nitrogen on growth and earheads density of wheat

\begin{tabular}{|c|c|c|c|c|c|c|c|c|c|c|c|c|c|c|c|}
\hline \multirow{2}{*}{$\begin{array}{l}\text { Treatment } \\
\text { equal splits }\end{array}$} & \multicolumn{5}{|c|}{ Plant Height, cm } & \multicolumn{5}{|c|}{ Earhead length, cm } & \multicolumn{5}{|c|}{ Earheads/sq. m. } \\
\hline & $\begin{array}{c}2010- \\
11\end{array}$ & $\begin{array}{c}2011- \\
12\end{array}$ & $\begin{array}{c}2012- \\
13\end{array}$ & $\begin{array}{c}2013- \\
14\end{array}$ & Pooled & $\begin{array}{c}2010- \\
11\end{array}$ & $\begin{array}{c}2011- \\
12\end{array}$ & $\begin{array}{c}2012- \\
13\end{array}$ & $\begin{array}{c}2013- \\
14\end{array}$ & Pooled & $\begin{array}{c}2010- \\
11\end{array}$ & $\begin{array}{c}2011- \\
12\end{array}$ & $\begin{array}{c}2012- \\
13\end{array}$ & $\begin{array}{c}2013- \\
14\end{array}$ & Pooled \\
\hline$-90 \mathrm{~N}, 3$ & 78.9 & 81.1 & 83.2 & 77.33 & 80 & 8.0 & 9.0 & 7.2 & 8.8 & 8.3 & 454 & 361 & 409 & 341 & 391 \\
\hline T2-90N, 4 aft & 70.9 & 74.9 & 80.9 & 75.07 & 75.5 & 1.1 & 9.2 & 7.3 & 8.3 & 8.1 & 401 & 358 & 400 & 345 & 391 \\
\hline Т3-90 & 78.7 & 84.3 & 79.6 & 78.47 & 80 & 78 & 8.9 & 7.7 & 8 & 8.1 & 483 & 368 & 422 & 394 & 416 \\
\hline T4-9 & 76.5 & 83.3 & 81.9 & 80.33 & 80 & 7.9 & 9.3 & 7.9 & 7.9 & 8.2 & 47 & 362 & 428 & 359 & 405 \\
\hline T5-120N, 3 & 77.3 & 84.6 & 82.7 & 85.27 & 82. & 7.5 & 8.9 & 7.7 & 8.8 & 8 & 470 & 423 & 458 & 375 & 432 \\
\hline $\mathrm{T} 6-1$ & 74 & 7 & 8 & 75. & 77.9 & 7.5 & 9.2 & 7.4 & 8.1 & & 45 & 38 & 46 & 3 & 413 \\
\hline T7-120N, 3 & 80.0 & 85.0 & 85.8 & 77.47 & 82.1 & 8.3 & 9.1 & 7.5 & 8.5 & 8.4 & 533 & 433 & 480 & 437 & 470 \\
\hline $\mathrm{T} 8-1$ & 78 & 8 & 84.1 & 81.47 & 8 & 7.4 & 9. & 7.5 & 8.4 & & 4 & 3 & 48 & 4 & 43 \\
\hline T9-150N, 3 & 77.9 & 85.9 & 85.2 & 83.33 & 83.1 & 8.1 & 9.3 & 7.5 & 8.5 & 8.3 & 496 & 455 & 474 & 415 & 460 \\
\hline T10-150N, 4 after irrigation & 74.8 & 85.5 & 84.5 & 86.60 & 82.9 & 7.5 & 9.3 & 8.1 & 8.5 & 8.4 & 483 & 375 & 490 & 421 & 442 \\
\hline T11-150N, 3 before & 80.8 & 90.4 & 84.3 & 82.87 & 84.6 & 7.7 & 9.3 & 7.5 & 8.3 & 8.2 & 516 & 473 & 489 & 476 & 488 \\
\hline $\mathrm{T} 12-150 \mathrm{~N}, 4$ & 79.0 & 91.5 & 83.6 & 82.93 & 84.3 & 8.1 & 9.2 & 7.9 & 8.6 & 8. & 509 & 450 & 495 & 443 & 474 \\
\hline T13-Control (no nitrogen) & 60.1 & 59.6 & 63.0 & 49.57 & 58.1 & 6.2 & 7.5 & 6.4 & 7.2 & 6.8 & 319 & 303 & 291 & 297 & 303 \\
\hline
\end{tabular}


among different levels, time of application of urea and number of splits of nitrogen were found at par among themselves (Table 2) indicting no effect of timing and split application on earhead length.

Earhead density was also significantly affected by the application of nitrogen levels and all the nitrogen levels levels of nitrogen and it was numerically better than four splits and urea top dressed after irrigation during all the years as well as on pooled basis (Table 2).

Perusal of the data presented in Table 3 revealed that levels, time and splits of nitrogen did not influence the boldness of the wheat grain (1000 grains weight,) during all

Table 3. Effect of pre and post irrigation and split application of urea nitrogen on yield attributes and biomass of wheat

\begin{tabular}{|c|c|c|c|c|c|c|c|c|c|c|c|c|c|c|c|}
\hline \multirow{2}{*}{$\begin{array}{l}\text { Treatment } \\
\text { equal splits, }\end{array}$} & \multicolumn{5}{|c|}{1000 grains weight, $g$} & \multicolumn{5}{|c|}{ Number of grains/ Earhead } & \multicolumn{5}{|c|}{ Biomass, q ha-1 } \\
\hline & $\begin{array}{c}2010- \\
11\end{array}$ & $\begin{array}{c}2011- \\
12\end{array}$ & $\begin{array}{c}2012- \\
13\end{array}$ & $\begin{array}{c}2013- \\
14\end{array}$ & Pooled & $\begin{array}{c}2010- \\
11\end{array}$ & $\begin{array}{c}2011- \\
12\end{array}$ & $\begin{array}{c}2012- \\
13\end{array}$ & $\begin{array}{c}2013- \\
14\end{array}$ & Pooled & $\begin{array}{c}2010- \\
11\end{array}$ & $\begin{array}{c}2011- \\
12\end{array}$ & $\begin{array}{c}2012- \\
13\end{array}$ & $\begin{array}{c}2013- \\
14\end{array}$ & Pooled \\
\hline T1-90N, 3 after irrigation & 41.37 & 38.11 & 37.23 & 37.12 & 38.46 & 26.07 & 33.71 & 29.54 & 33.06 & 30.59 & 114.29 & 108.33 & 107.14 & 96.83 & 106.65 \\
\hline T2-90N, 4 after irrigation & 40.55 & 37.39 & 3765 & 3656 & 4 & 22.55 & 27.25 & 0903 & 30.06 & 27.22 & 94.44 & 0107 & 103.97 & 5.71 & 93.85 \\
\hline T3-90N, 3 before irrigation & 39.60 & 38.42 & 37.35 & 37.31 & 38.17 & 28.66 & 34.13 & 30.16 & 29.06 & 30.50 & 116.27 & 114.68 & 113.49 & 101.59 & 111.51 \\
\hline T4-90N, 4 before irrigation & 40.26 & 38.10 & 36.73 & 36.59 & 37.92 & 24.05 & 32.55 & 29.48 & 31.11 & 29.30 & 101.59 & 107.54 & 112.30 & 92.46 & 103.47 \\
\hline T5-120N, 3 afte & 40.10 & 39.49 & 37.80 & 37.97 & 38.84 & 27.98 & 27.46 & 27.30 & 32.33 & 28.77 & 114.29 & 114.68 & 114.29 & 103.57 & 111.71 \\
\hline T6-120N, 4 a & 40.77 & 38.12 & 36.45 & 37.05 & 38.10 & 27.11 & 28.78 & 28.71 & 32.20 & 29.20 & 105.16 & 104.76 & 115.48 & 92.46 & 104.46 \\
\hline T7-120N, 3 before irrigation & 39.25 & 38.31 & 37.39 & 37.25 & 38.05 & 26.88 & 31.88 & 28.06 & 29.00 & 28.95 & 127.78 & 124.21 & 119.44 & 109.52 & 120.24 \\
\hline T8-120N, 4 before irrigation & 39.71 & 39.11 & 37.07 & 36.80 & 38.17 & 25.27 & 34.30 & 28.10 & 29.14 & 29.20 & 117.06 & 125.79 & 118.65 & 98.02 & 114.88 \\
\hline T9-150N, 3 after irrigation & 39.12 & 38.55 & 37.07 & 36.94 & 37.92 & 29.34 & 30.48 & 29.92 & 32.72 & 30.62 & & 126.59 & 123.02 & 113.10 & 121.73 \\
\hline T10-150N, 4 after irrigation & 40.97 & 38.03 & 36.58 & 37.36 & 38.24 & 26.71 & 35.68 & 28.86 & 29.93 & 30.30 & 113.89 & 122.22 & 123.81 & 100.79 & 115.18 \\
\hline T11-150N, 3 before irrigation & 39.12 & 38.36 & 37.24 & 36.99 & 37.93 & 30.90 & 33.64 & 29.92 & 31.22 & 31.42 & 141.67 & 142.06 & 126.98 & 123.41 & 133.53 \\
\hline T12-150N, 4 before irrigation & 40.11 & 37.93 & 36.99 & 37.26 & 38.07 & 29.19 & 34.48 & 29.42 & 30.87 & 30.99 & 134.92 & 136.51 & 128.17 & 111.90 & 127.88 \\
\hline T13-Control (no nitrogen) & 37.36 & 37.43 & 37.09 & 36.25 & 37.03 & 17.38 & 18.85 & 23.32 & 17.26 & 19.20 & 47.22 & 45.24 & 57.54 & 44.05 & 48.51 \\
\hline $\mathrm{CD}(0.05)$ & 1.52 & NS & NS & NS & NS & 2.78 & 2.83 & 2.53 & 2.44 & 3.04 & 4.80 & 4.90 & 5.84 & 4.83 & 4.99 \\
\hline
\end{tabular}

recorded significantly higher earhead density than control (no nitrogen).On pooled basis, highest earhead density (488/ sq. m.) was recorded in treatment where $150 \mathrm{~kg} \mathrm{~N}$ $\mathrm{ha}^{-1}$ was applied in three splits just before irrigation and it was significantly higher than the $90 \mathrm{~kg} \mathrm{~N} \mathrm{ha-1}$ applied in three or four splits just before or after irrigation (Table 2). Three split application of $\mathrm{N}$ as urea top dressed just before irrigation recorded more earhead density at all the the years of study as well as on pooled basis except during the year 2010-11 where all the nitrogen levels, times and splits produced significantly bolder grains than control.

Number of grains/earhead was also significantly influenced by levels, time of $\mathrm{N}$ application as well as number of splits as compared to control (no nitrogen). The highest numbers of grains/earhead (31.42 grains/earhead) on pooled basis were recorded in treatment where $150 \mathrm{~kg} \mathrm{~N}$ was applied

Table 4. Effect of pre and post irrigation and split application of urea nitrogen on yield and quality of wheat

\begin{tabular}{|c|c|c|c|c|c|c|c|c|c|c|c|c|c|c|c|}
\hline \multirow{2}{*}{$\begin{array}{l}\text { Treatment } \\
\text { equal splits, }\end{array}$} & \multicolumn{5}{|c|}{ Grain yield, q ha-1 } & \multicolumn{5}{|c|}{ Straw yield, q ha-1 } & \multicolumn{5}{|c|}{ Grain protein content, $\%$} \\
\hline & $\begin{array}{c}2010- \\
11\end{array}$ & $\begin{array}{c}2011- \\
12\end{array}$ & $\begin{array}{c}2012- \\
13\end{array}$ & $\begin{array}{c}2013- \\
14\end{array}$ & Pooled & $\begin{array}{c}2010- \\
11\end{array}$ & $\begin{array}{c}2011- \\
12\end{array}$ & $\begin{array}{c}2012- \\
13\end{array}$ & $\begin{array}{c}2013- \\
14\end{array}$ & Pooled & $\begin{array}{c}2010- \\
11\end{array}$ & $\begin{array}{c}2011- \\
12\end{array}$ & $\begin{array}{c}2012- \\
13\end{array}$ & $\begin{array}{c}2013- \\
14\end{array}$ & Pooled \\
\hline T1-90N, 3 after irrigation & 49.00 & 46.36 & 44.92 & 41.81 & 45.52 & 65.29 & 61.97 & 62.22 & 55.02 & 61.13 & 10.34 & 9.20 & 10.57 & 11.63 & 10.44 \\
\hline T2-90N, 4 after irrigation & 42.12 & 36.52 & 43.69 & 37.90 & 40.06 & 52.32 & 54.75 & 60.27 & 47.82 & 53.79 & 10.14 & 9.37 & 10.07 & 10.30 & 9.97 \\
\hline T3-90N, 3 before irrigation & 54.67 & 48.05 & 47.42 & 42.73 & 48.22 & 61.60 & 66.63 & 66.07 & 58.86 & 63.29 & 9.90 & 9.30 & 10.03 & 11.37 & 10.15 \\
\hline T4-90N, 4 before irrigation & 45.64 & 44.77 & 46.28 & 40.88 & 44.39 & 55.94 & 62.77 & 66.02 & 51.58 & 59.08 & 10.07 & 9.67 & 10.30 & 10.60 & 10.16 \\
\hline T5-120N, 3 after irrigation & 52.71 & 45.85 & 47.28 & 46.00 & 47.96 & 61.58 & 68.83 & 67.00 & 57.58 & 63.75 & 10.03 & 9.10 & 10.73 & 12.23 & 10.52 \\
\hline T6-120N, 4 after irrigation & 49.25 & 42.18 & 48.15 & 42.25 & 45.46 & 55.91 & 62.58 & 67.33 & 50.21 & 59.01 & 10.19 & 9.33 & 10.80 & 11.40 & 10.43 \\
\hline T7-120N, 3 before irrigation & 56.07 & 52.79 & 50.31 & 47.17 & 51.59 & 71.71 & 71.41 & 69.13 & 62.35 & 68.65 & 9.81 & 9.23 & 10.67 & 12.07 & 10.45 \\
\hline T8-120N, 4 before irrigation & 49.67 & 50.17 & 50.17 & 43.43 & 48.36 & 67.40 & 75.63 & 68.48 & 54.59 & 66.52 & 9.93 & 9.37 & 10.83 & 10.73 & 10.22 \\
\hline T9-150N, 3 after irrigation & 56.83 & 53.46 & 52.61 & 50.15 & 53.26 & 67.38 & 73.13 & 70.40 & 62.95 & 68.47 & 9.78 & 9.67 & 10.77 & 12.00 & 10.55 \\
\hline T10-150N, 4 after irrigation & 52.75 & 50.76 & 51.73 & 46.97 & 50.55 & 61.14 & 71.46 & 72.08 & 53.82 & 64.63 & 10.24 & 9.50 & 11.73 & 11.47 & 10.74 \\
\hline T11-150N, 3 before irrigation & 62.25 & 60.94 & 54.50 & 54.92 & 58.15 & 79.42 & 81.13 & 72.49 & 68.50 & 75.38 & 9.78 & 9.53 & 11.63 & 12.73 & 10.92 \\
\hline T12-150N, 4 before irrigation & 59.63 & 58.81 & 53.81 & 50.82 & 55.77 & 75.29 & 77.70 & 74.37 & 61.08 & 72.11 & 10.03 & 9.97 & 11.73 & 11.50 & 10.81 \\
\hline T13-Control (no nitrogen) & 20.69 & 21.38 & 25.08 & 18.50 & 21.41 & 26.53 & 23.86 & 32.46 & 25.55 & 27.10 & 9.34 & 9.37 & 9.90 & 9.92 & 9.63 \\
\hline $\mathrm{CD}(0.05)$ & 2.40 & 2.61 & 1.51 & 2.54 & 2.09 & 4.64 & 4.28 & 7.58 & 3.58 & 5.01 & 0.38 & NS & 0.86 & 0.83 & 0.67 \\
\hline
\end{tabular}


in three splits just before irrigation indicating the positive effect of nitrogen top dressed just before irrigation (Table 3). The significantly lowest numbers of grains/earhead (19.20 grains/earhead) on pooled basis were recorded in treatment where no nitrogen was applied.

The results revealed that the biomass production was influenced significantly by time, level and split application of urea top dressing (Table 3). Biomass was significantly higher in all the levels of nitrogen from 90 to $150 \mathrm{~kg}$ ha-1 than control treatment. Biomass was also significantly higher where urea was top dressed just before irrigation than urea top dressed after irrigation at all the levels of nitrogen in three split application as compared to four split application on yearly as well as on pooled basis.

Grain yield of wheat was significantly affected by levels of nitrogen, split application and time of urea top dressing. The significantly higher (58.15 q ha-1) grain yield was observed in treatment where $\mathrm{N}$ at $150 \mathrm{~kg}$ ha- 1 was top dressed just before irrigation with three split application during all the years as well as on pooled basis (Table 4). As expected, the lowest grain was recorded in absolute control treatment. It was reported (Coventry et al., 2011) earlier also that three spit application of $150 \mathrm{~kg} \mathrm{~N} \mathrm{ha}{ }^{-1}$ (three equal splits as $1 / 3^{\text {rd }}$ basal, $1 / 3^{\text {rd }}$ at first irrigation at $25 \mathrm{DAS}$ and $1 / 3^{\text {rd }}$ at second irrigation at $45 \mathrm{DAS}$ ) were better than two split application in Haryana conditions.

The application of urea just before irrigation recorded higher grain yield at all the levels of nitrogen application than corresponding level where urea was top dressed after irrigation (about a week) indicating the positive impact of before irrigation urea top dressing and a yield gain of 2.70-5.21 q ha ${ }^{-1}$ was observed on pooled basis (Table 5). It indicates greater recovery of applied nitrogen due to top dressing just before irrigation which was reflected in consistently higher productivity.

Straw yield of wheat was significantly affected by levels of nitrogen, split application and time of urea top dressing. The highest (75.38 q ha ${ }^{-1}$ ) and significantly higher straw yield was observed in treatment where urea was top dressed just before irrigation with three split application of $\mathrm{N}$ at $150 \mathrm{~kg}$ ha-1 during all the years and on pooled basis. The lowest straw yield (27.10 q ha-1) was recorded in absolute control treatment (Table 4).

Wheat quality measured in the form of grain protein content was also significantly influenced by nitrogen levels and time of application (Table 4). All the levels of nitrogen recorded significantly higher grain protein content than absolute control indicating the positive effect of Nitrogen for achieving better quality of wheat. On pooled basis, it was observed that three split application of nitrogen and urea top dressing just before irrigation had higher grain protein content than four split application and urea top dressed after irrigation but the increase was numerical only (Table 4).

Agronomic nitrogen use efficiency (NUE) was also influenced by levels of $\mathrm{N}$, number of splits and time of urea top dressing. The highest agronomic NUE (29.8 kg grain $\mathrm{kg}^{-1} \mathrm{~N}$ applied) was recorded where $90 \mathrm{~kg} \mathrm{ha}^{-1} \mathrm{~N}$ was applied in three splits and urea was top dressed just before irrigation (Table 5). Urea top dressing just before irrigation brought about higher agronomic NUE than urea top dressed after irrigation in three split application (three equal splits as $1 / 3^{\text {rd }}$ basal, $1 / 3^{\text {rd }}$ at first irrigation at $20-25$ DAS and $1 / 3^{\text {rd }}$ at second irrigation at $40-45$ DAS) than four split application (as $1 / 4^{\text {th }}$ basal, $1 / 4^{\text {th }}$ at first irrigation at 20-25 DAS, 1/4th at second irrigation at 40-45 DAS and $1 / 4^{\text {th }}$ at third irrigation at $60-65$ DAS) at all the levels

Table 5. Effect of pre and post irrigation urea split application on agronomic NUE, yield gain and economic returns

\begin{tabular}{|c|c|c|c|c|}
\hline Treatment & $\begin{array}{l}\text { Agronomic NUE, } \\
\text { kg grain per kg } \\
\text { nitrogen applied }\end{array}$ & $\begin{array}{c}\text { Yield gain with } \\
\text { pre irrigation over } \\
\text { corresponding post } \\
\text { irrigation urea application }\end{array}$ & $\begin{array}{l}\text { Percent yield gain with } \\
\text { pre irrigation over } \\
\text { corresponding post } \\
\text { irrigation urea application }\end{array}$ & $\begin{array}{l}\text { Net economic returns } \\
\text { by applying pre } \\
\text { irrigation urea over post } \\
\text { irrigation (Rs. ha-1) }\end{array}$ \\
\hline T1- 90N, 3 equal splits, after irrigation & 26.79 & - & - & - \\
\hline T2- 90N, 4 equal splits, after irrigation & 20.72 & - & - & - \\
\hline T3- 90N, 3 equal splits, before irrigation & 29.78 & 2.70 & 5.93 & $4680^{*}$ \\
\hline T4- $90 \mathrm{~N}$, 4 equal splits, before irrigation & 25.53 & 4.34 & 10.82 & 7522 \\
\hline T5- $120 \mathrm{~N}, 3$ equal splits, after irrigation & 22.12 & - & - & - \\
\hline T6- $120 \mathrm{~N}, 4$ equal splits, after irrigation & 20.04 & - & - & - \\
\hline T7- $120 \mathrm{~N}, 3$ equal splits, before irrigation & 25.14 & 3.63 & 7.56 & 6295 \\
\hline T8- $120 \mathrm{~N}, 4$ equal splits, before irrigation & 22.45 & 2.90 & 6.38 & 5035 \\
\hline T9- $150 \mathrm{~N}, 3$ equal splits, after irrigation & 21.23 & - & - & - \\
\hline T10- $150 \mathrm{~N}, 4$ equal splits, after irrigation & 19.43 & - & - & - \\
\hline T11- $150 \mathrm{~N}, 3$ equal splits, before irrigation & 24.49 & 4.89 & 9.18 & 8481 \\
\hline T12- $150 \mathrm{~N}, 4$ equal splits, before irrigation & 22.90 & 5.21 & 10.31 & 9043 \\
\hline
\end{tabular}


of nitrogen i.e. 90 to $150 \mathrm{~kg} \mathrm{ha}^{-1}$. It clearly indicates that three split application of $\mathrm{N}$ and top dressing of urea just before irrigation is the best nitrogen management strategy for irrigated wheat under North Indian conditions. The practice of urea top dressing just before irrigation resulted in 2.70-5.21q ha-1 (average $3.94 \mathrm{q} \mathrm{ha}^{-1}$ ) additional yield compared to respective treatment of urea top dressing after irrigation (Table 5). This yield gain was 5.93 to 10.83 percent (average $8.36 \%$ ) higher yield than urea top dressed after irrigation which resulted in additional benefit of Rs. 4680 to 9043 ha $^{-1}$ (average Rs. 6843 ha $^{-1}$ ) at the wheat MSP of Rs. $1735 / \mathrm{q}^{-1}$ (Table 5).

Three split application of $\mathrm{N}$ and urea top dressed just before irrigation treatments were found better than four split application of $\mathrm{N}$ and urea top dressed after irrigation at all the levels of $\mathrm{N}$ in improving the growth, yield attributes, yield and quality of wheat crop as well as in improving agronomic NUE which might be due the reason that $\mathrm{N}$ applied through urea top dressed just before irrigation was efficiently dissolved in the irrigation water and stored in the root zone of the wheat crop and thus reduced the volatilization losses of N. Earlier, Katyal et al. (1987) also reported that the apparent recovery $\mathrm{N}$ through urea applied just before irrigation was higher $(62 \%)$ than the apparent recovery of $\mathrm{N}$ through urea applied after irrigation. Similarly,Fernando Viero et al. (2015) also reported that sprinkler irrigation immediately after $\mathrm{N}$ fertilization is effective in reducing ammonia losses by volatilization, particularly from urea.

\section{Conclusion}

On the basis of this four year study it can be concluded that $150 \mathrm{~kg} \mathrm{~N} \mathrm{ha}{ }^{-1}$ applied in three equal splits (as $1 / 3^{\text {rd }}$ basal, $1 / 3^{\text {rd }}$ at first irrigation at $20-25$ DAS and $1 / 3^{\text {rd }}$ at second irrigation at 40-45 DAS) with top dressing using urea just before irrigation was the most efficient practice of nitrogen management for better growth, yield and quality of wheat as well as better recovery of applied $\mathrm{N}$ along with greater profitability to farmers.

\section{References}

1. Alam MM, JK Ladha, Foyjunnessa, Z Rahman, SR Khan, Harun-ur-Rashid, AH Khan andRJ Buresh. 2006. Nutrient management for increased productivity of rice-wheat cropping system in Bangladesh. Field Crops Research 96: 374-386.

2. Singh B, RK Sharma, J Kaur, ML Jat, KL Martin, Y Singh, V P Singh, P Chandna, OP Choudhary, RK Gupta, HS Thind, J Singh, HS Uppal, HS Khurana, A Kumar, RK Uppal, M Vashistha, WR Raun and R Gupta. 2011. Assessment of nitrogen management strategy using an optical sensor for irrigated wheat. Agronomy for Sustainable Development 31:589-603.

3. Bhardwaj V, V Yadav and BS Chauhan. 2010. Effect of nitrogen application timings and varieties on growth and yield of wheat grown on raised beds. Archives of Agronomy and Soil Science. 56: 211-222.

4. Coventry DR, A Yadav, RS Poswal, RKSharma, RK Gupta, RS Chhokar, SC Gill, V Kumar, A Kumar, A Mehta, SGL Kleemann, and JA Cummins. 2011. Irrigation and nitrogen scheduling as a requirement for optimising wheat yield and quality in Haryana, India. Field Crops Research 123: 80-88.

5. Fernando Viero, C Bayer, RC Beber Vieira and Eduardo Carniel. 2015. Management of irrigation and nitrogen fertilizers to reduce ammonia volatilization. Revista Brasileira de Cienciado Solo 39:1737-1743.

6. Hobbs PR, KD Sayre and JI Ortiz-Monasterio. 1998. Increasing wheat yields sustainably through agronomic means. NRG Paper 98-01. Mexico, D.F., Mexico.

7. Katyal JC, B Singh, PLG Vlek and RJ Buresh. 1987. Efficient nitrogen use as affected by urea application and irrigation sequence. Soil Science Society of America Journal 51:366-370.

8. Mishra B, J Shoran, R Chatrath, AK Chatrath, RK Sharma, RK Gupta, R Singh, J Rane and A Kumar 2005. Cost effective and sustainable wheat production technologies. Directorate of Wheat Research, Karnal-132001, Technical Bulletin No. 8, p. 36.

9. Raun WR and GV Johnson. 1999. Improving nitrogen use efficiency for cereal production. Agronomy Journal 91: 357-363.

10. Singh S, RK Malik, JS Dhankar, A Yadav, G Rajbir, BR Kamboj, P Sheoran, and OP Lathwal. 2010. Nutrient use pattern in the irrigated rice-wheat cropping system in the Indo-Gangetic plains of Haryana, India. Experimental Agriculture 46: 191-209.

11. Srivastava S, AS Rao, K Alivelu, KN Singh, NS Raju and A Rathore. 2006. Evaluation of crop responses to applied fertilizer phosphorus and derivation of optimum recommendations using the MitscherlichBray equation. Communication in Soil Science and Plant Analysis 37: 847-858.

12. Woolfolk CW, WR Raun, GV Johnson, WE Thomason, RW Mullen, KJ Wynn and KW Freeman 2002. Influence of late-season foliar nitrogen applications on yield and grain nitrogen in wheat. Agronomy Journal 94: 429-434. 